\title{
Cirugía miniinvasiva transanal (TAMIS). Una alternativa para la resección de una cicatriz rectal luego de una resección endoscópica insuficiente
}

\author{
Alejandra Liz, Isabel Gabito, Álvaro Lavega, Emilia Cerchiari, Gonzalo \\ Gayo, Diego Lapiedra, Marcelo Viola \\ Unidad de Coloproctología. Clínica Quirúrgica “1” Hospital Pasteur. Prof. Dr. Fernando \\ González Calcagno. Montevideo, Uruguay.
}

\begin{abstract}
RESUMEN
La resección transanal miniinvasiva ha mejorado los resultados de las resecciones transanales clásicas. Estas técnicas se han difundido en los últimos años debido al desarrollo del TAMIS (trans anal minimally invasive surgery), que consiste en un puerto multicanal transanal por el que se introduce instrumental laparoscópico clásico. Debido a esto, no solo ha aumentado el número de procedimientos transanales realizados, sino también sus indicaciones, incluyendo un amplio abanico de patologías.

En esta publicación destacamos su rol como procedimiento miniinvasivo de exéresis de un adenocarcinoma rectal insuficientemente resecado luego de un procedimiento endoscópico, permitiendo un estudio adecuado de la lesión. Se describirán los principales detalles de la técnica y sus resultados.
\end{abstract}

Palabras claves: Cirugía transanal; TAMIS; Pólipos colorrectales

\section{ABSTRACT}

Transanal minimally invasive resection has improved the results of classic transanal resections. These techniques have spread in recent years due to the development of TAMIS (transanal minimally invasive surgery), which consists of a transanal multichannel port through which classic laparoscopic instruments are introduced. Due to this, not only the number of transanal procedures performed has increased, but also their indications, including a wide range of conditions.

In this publication we highlight its role as a minimally invasive procedure for the excision of an insufficiently resected rectal adenocarcinoma after an endoscopic procedure, allowing an adequate study of the lesion. The main details of the technique and its results will be described.

Keywords: Transanal Surgery; Tamis; Colorectal Polyps

\section{INTRODUCCIÓN}

El equilibrio para lograr un tratamiento adecuado y mantener la calidad de vida ha derivado en el desarrollo de nuevas técnicas y tecnologías. Las técnicas transanales combinadas con el abordaje endoscópico surgen como alternativa a la resección local convencional. ${ }^{1}$

En 1988, Buess et al., ${ }^{2}$ describen la microcirugía endoscópica transanal (TEM: transanal endoscopic microsurgery) como ejemplo de cirugía por orificios naturales y posteriormente surge la cirugía endoscópica transanal (TEO: transanal endoscopic operation). Estas técnicas permitieron mejorar la visibilidad y calidad de la resección, así como tratar lesiones en todos los sectores del recto y sigmoides distal, con mejores resultados que la cirugía transanal convencional. ${ }^{3}$

E1 TAMIS (trans anal minimally invasive surgery) surge a partir del desarrollo de la cirugía mininvasiva de

Los autores declaran ausencia de conflictos de interés.

Marcelo Viola Malet

mviolam@gmail.com

Recibido: septiembre de 2020. Aceptado: mayo de 2021. puerto único (single port). Descrita en 2010 por Atallah et al. ${ }^{4}$ como una alternativa al TEM/TEO, consiste en la utilización de un puerto único multicanal transanal combinado con el uso de instrumental laparoscópico. ${ }^{5}$ Esto ha facilitado su desarrollo y ampliado las indicaciones de lesiones pasibles de resección transanal incluyendo: lesiones benignas, particularmente los pólipos; ${ }^{6,7}$ tumores neuroendócrinos; ${ }^{7}$ tumores del estroma gastrointestinal (GIST) ; ${ }^{1}$ resección con criterio curativo de adenocarcinomas rectales $\mathrm{T} 1$ (habitualmente $1 / 4$ de circunferencia, bien diferenciados, sin angio/linfoinvasión); ; ${ }^{1,6,7}$ resección de tumores T2, siendo una indicación controversial ya que existe riesgo de metástasis ganglionares (puede considerarse en T2 bien diferenciados, sin invasión vascular/linfática y, en estos casos, la evidencia recomienda fuertemente la necesidad de tratamiento adyuvante, quimiorradiante); ${ }^{1,6,7}$ resecciones no oncológicas en pacientes con elevado riesgo quirúrgico o por elección propia; y exéresis biópsica de la "cicatriz" de una lesión rectal luego del tratamiento neoadyuvante. ${ }^{1}$ En este caso, destacamos su rol como coadyuvante de la resección endoscópica frente a resecciones insuficientes (superficiales, márgenes 


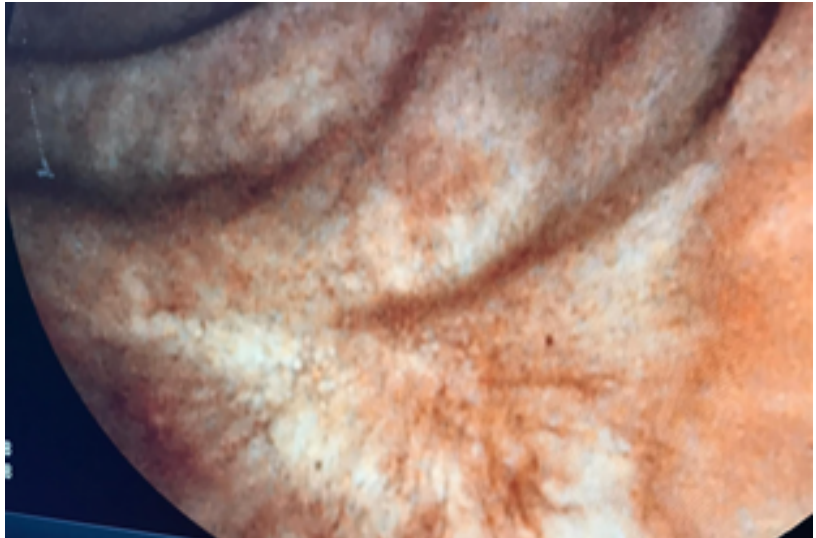

Figura 1: Se observa la cicatriz de aspecto nacarado, con fibrosis convergente.

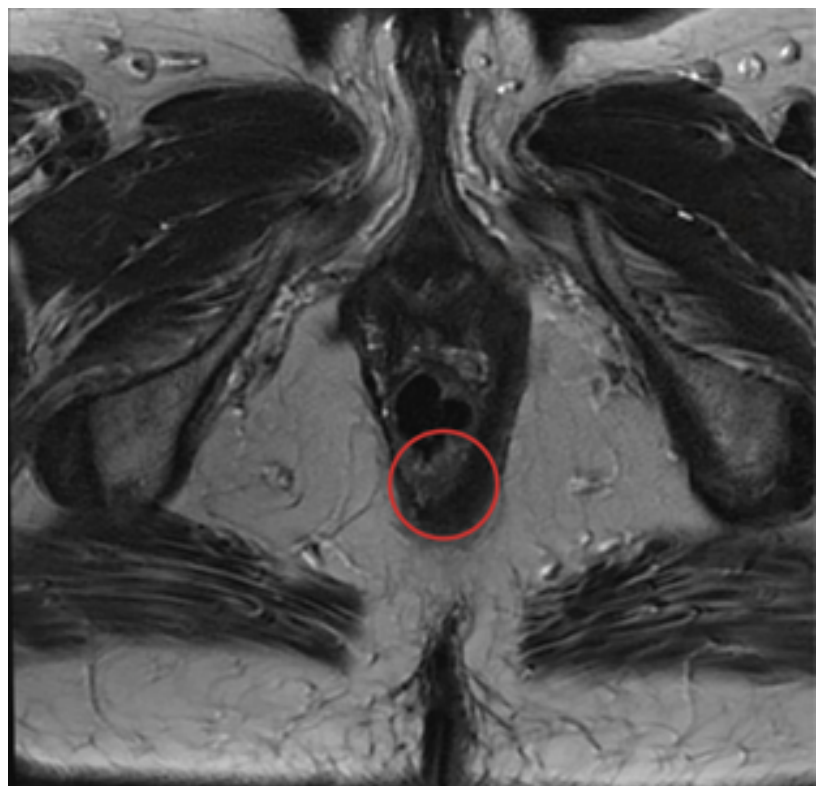

Figura 2: Obsérvese el engrosamiento mucoso en cara pósterolateral izquierda.

insuficientes, fragmentadas).

Menos frecuentemente, se ha descrito su uso para la reparación de fístulas rectoureterales, hemostasis de lesión de Dielafoy rectal, reparación de fallas de suturas colo o ileorrectales, extracción de cuerpos extraños y abordajes transcolostomía. ${ }^{9}$

\section{MÉTODO}

Se presenta el caso de una paciente de 68 años a la que se le resecó endoscópicamente, durante una videocolonoscopia, un polipo rectal sésil a $7 \mathrm{~cm}$ del margen anal $\left(\mathrm{Fi}^{-}\right.$ gura 1). El informe histológico refleja la resección endoscópica incompleta de un adenocarcinoma de recto bajo, con infiltración submucosa de $4 \mathrm{~mm}$ de profundidad y márgenes de resección comprometidos. Para completar la valoración oncológica del mesorrecto se realizó una resonancia nuclear magnética de recto (Figura 2) que descarta compromiso ganglionar mesorrectal, así como hace

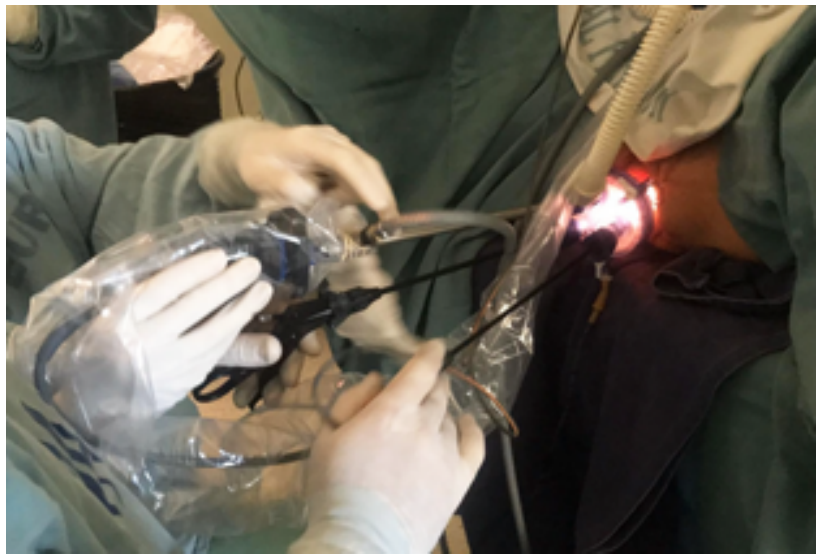

Figura 3: Colocación de la plataforma y comienzo de la cirugía.

referencia a una alteración de la pared del recto, probablemente vinculada a una cicatriz. La evaluación de lesiones a distancia se realizó con tomografía de tórax, abdomen y pelvis y marcadores tumorales los cuales fueron normales. La estadificación cTNM fue T1N0M0, por lo que se decidió la resección de espesor parietal total por TAMIS para estudio completo de la lesión. No se hizo una ecografía endorrectal, ya que no tenemos disponibilidad permanente de dicho estudio.

El procedimiento se realizó bajo anestesia general; con ayuno previo, preparación retrógrada del recto para la correcta visualización de la lesión y profilaxis antibiótica con Ampicilina-Sulbactam.

La paciente se colocó en posición ginecológica y Trendelemburg optimizando la visión de la luz rectal.

Se colocaron el monitor hacia la cabeza del paciente, el cirujano entre las piernas a la derecha y el asistente a la izquierda.

Tacto rectal sin lesiones. Esfínter normotónico.

Dilatación anal suave para facilitar la colocación del dispositivo y disminuir el riesgo de trauma esfintérico.

Introducción del GelPOINT Path ${ }^{\circledR}$ plegado, lubricado. Fijación a la piel para evitar su rotación o expulsión, minimizando el trauma del canal anal (Figura 3).

Colocación de la tapa hermética de gel y los tres puertos de acceso. En el puerto central se colocó la óptica y en los otros dos, los instrumentos laparoscópicos. El insuflador se conecta al trócar más superior para evitar que el flujo de $\mathrm{CO} 2$ "salpique" la óptica con el líquido acumulado en el sector declive del recto. Conexión del GelPOINT Path $^{\circledR}$ a una bolsa de estabilización de gas para mantener una cavidad estable. Neumorrecto a $12 \mathrm{mmHg}$. Identificación de cicatriz de polipectomía en cara póstero-lateral derecha, a $7 \mathrm{~cm}$ del margen anal. Realce de la lesión con azul de Metileno (Figura 4). Marcado circunferencial de la misma con electrobisturí (margen de $1 \mathrm{~cm}$ ). La resección comenzó a la hora 6 avanzando por ambos la- 


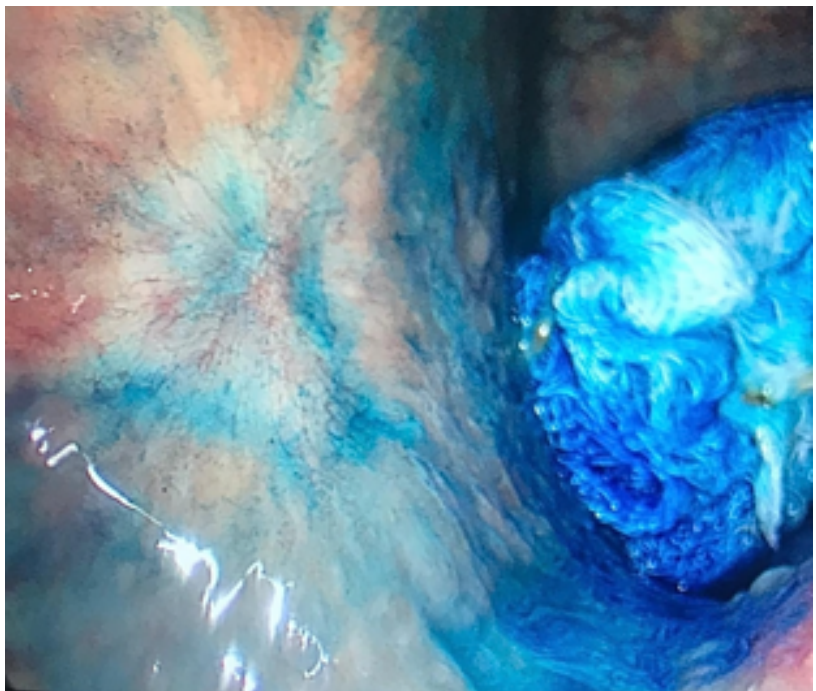

Figura 4: Realce de la lesión con azul de Metileno

dos hasta el sector proximal. Esta maniobra facilita la disección del margen en profundidad. Se tomó la pieza por mucosa sana para evitar su fragmentación, asegurando una pieza única y con margen adecuado (Figura 5). Extracción de la lesión retirando la tapa del dispositivo. Se repera para estudio anatomopatológico (Figura 6).

Cierre de la brecha rectal con sutura barbada 3-0 (VLocTM, Covidien, Mansfield, MA) (Figura 7).

Postoperatorio sin incidentes. Alta el mismo día.

Resultado anatomopatológico: cicatriz inflamatoria sin malignidad.

\section{DISCUSIÓN}

Frente al TEM/TEO, el abordaje TAMIS ha presentado ciertas ventajas: menor costo; plataforma más fácil de colocar; uso de instrumental laparoscópico; campo visual de $360^{\circ}$ (vs. $220^{\circ}$ del TEM); no requiere cambios de posición del paciente (con posibilidad de abordaje intraperitoneal combinado) y curva de aprendizaje corta para cirujanos expertos en laparoscopía. . $^{1,-5,8}$

Actualmente, existe un renovado interés en TAMIS debido al desarrollo de la escisión mesorrectal total transanal (taTME) combinado con resección laparoscópica intraabdominal, facilitando la escisión completa del mesorrecto, cuya disección es dificultosa por vía abdominal exclusiva ${ }^{8,10}$ En esta publicación, comunicamos también su valor en la resección de cicatrices rectales de procedimientos endoscópicas insuficientes.

En TAMIS, las dos plataformas más utilizadas son: SILS port ${ }^{\circledR}$ (Covidien, Mansfield, Massachusetts, EEUU) y GelPoint Path ${ }^{\circledR}$ (Applied Medical, Rancho Santa Margarita, California, EEUU). ${ }^{6}$ Su material flexible basado en un elastómero termoplástico permite ajus-

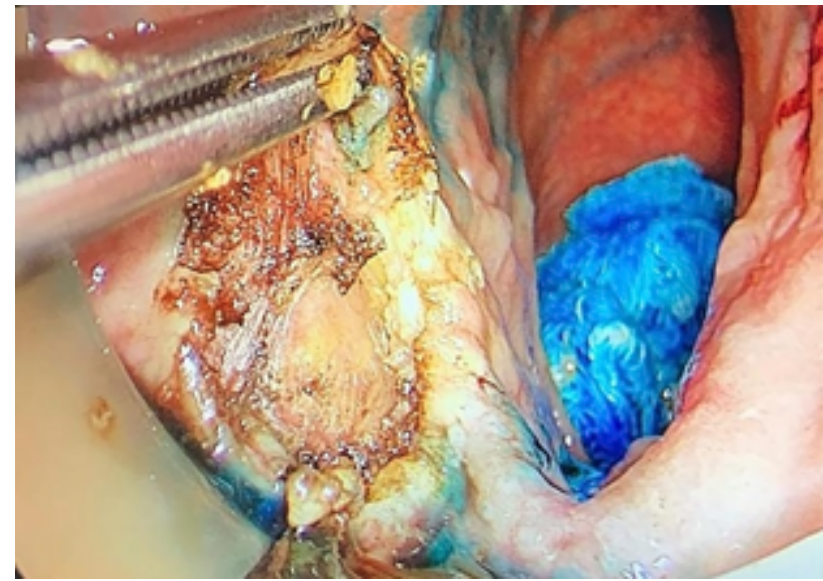

Figura 5: Comienzo de la disección.

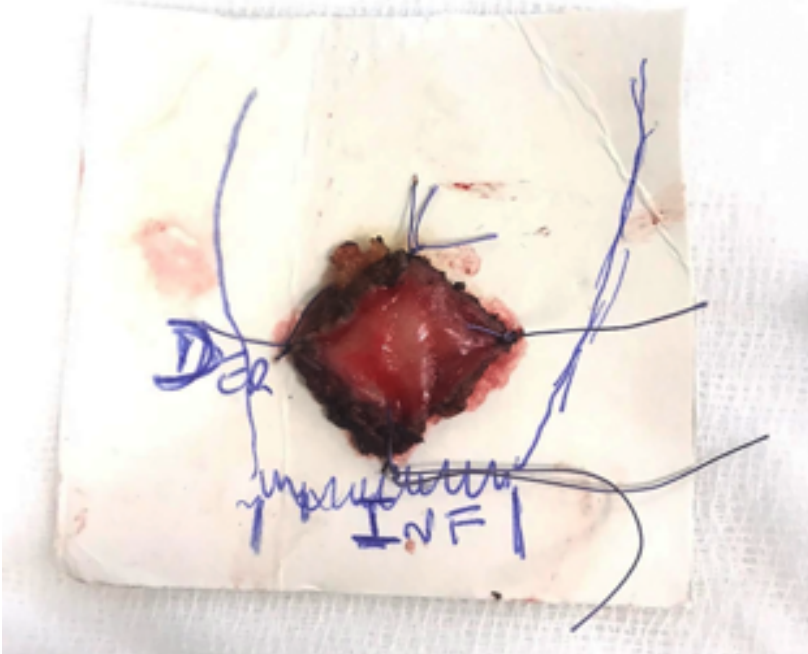

Figura 6: Resección completa. Marcado de la pieza para anatomía patológica.

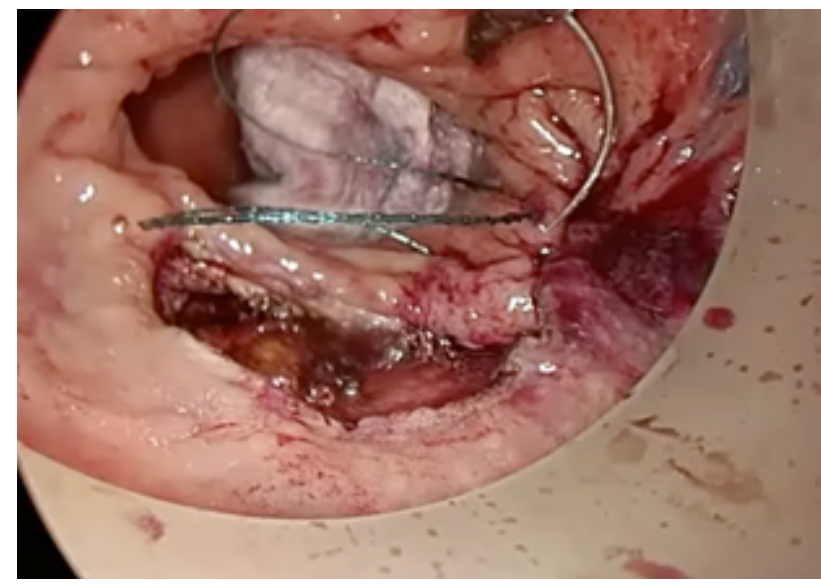

Figura 7: Cierre de la brecha rectal con sutura barbada 3-0.

tar instrumentos laparoscópicos de diferente tamaño. Se adapta al canal anal disminuyendo la distensión del esfínter y, gracias al material de confección, genera un sistema de sellado que minimiza la pérdida de $\mathrm{CO} 2$. La menor longitud en comparación al TEM, permite mayor 
angulación y movimiento de las pinzas durante el procedimiento. ${ }^{4,9}$

Una de las dificultades técnicas relacionadas al TAMIS es la inestabilidad del neumorrecto por el flujo pulsátil del insuflador. En los últimos años se han desarrollado dispositivos como el insuflador AirsealTM y las bolsas estabilizadoras de gas, como la que hemos utilizado. Esto crea un neumorrecto estable, evitando el flujo rítmico y el colapso de la luz rectal, facilitando el procedimiento. ${ }^{11} \mathrm{El}$ neumorrecto suele realizarse a $15 \mathrm{mmHg}$ con flujo alto, para lograr una adecuada distensión. ${ }^{8}$

Para la resección con márgenes adecuados, destacamos la utilidad del realce de la lesión con tinción vital como el azul de Metileno. ${ }^{12}$ Esto permite diferenciar mejor el área patológica de la mucosa normal, facilitando el marcado de la lesión con un margen seguro de $5-10 \mathrm{~mm} .{ }^{3} \mathrm{La}$ profundidad de la resección dependerá del tipo de lesión. En las resecciones anteriores de espesor parietal completo debe tenerse cuidado de no lesionar la vagina, la uretra/próstata. Es preferible la utilización del electrobisturí para visualizar los planos de disección, si bien pueden utilizarse otros métodos de hemostasis. La pieza obtenida debe ser única y marcarse adecuadamente para su estudio anatomopatológico. ${ }^{8}$

Un punto controversial es el cierre de la brecha rectal a nivel subperitoneal. Las últimas publicaciones recomiendan que debe intentarse siempre que sea posible. ${ }^{8}$ En cirujanos experimentados y centros de alto volumen se observa una tendencia a realizar el cierre parietal. Puede utilizarse una sutura barbada 2-0 o 3-0 que facilita la maniobra. ${ }^{3}$ Como ventajas, habría menos complicaciones fundamentalmente hemorrágicas y aumentaría la velocidad de cicatrización. Sin embargo, esto no está completamente demostrado y el cierre puede ser dificultoso. ${ }^{8}$

En cuanto a los resultados, el TAMIS tiene resultados similares a otras técnicas endoscópicas de cirugía transanal. ${ }^{1,10}$ Los porcentajes de resección incompleta o de fragmentación varían según las series, pero son mejores en comparación a la resección local clásica. Se reportan mayores márgenes de resección R0 (88-90 \% para la resección endoscópica vs. $55 \%$ local clásica), menor frag- mentación (1,4\% vs. $24 \%)$ y menor recurrencia local acumulada (4-6\% a $20 \%$ vs. $29 \%)^{1,3,4}$

La morbilidad global con las resecciones endoscópicas transanales varía entre el 7 y $31 \%$ en distintos reportes. ${ }^{3}$ Esta variabilidad depende de los criterios utilizados para su evaluación. Afortunadamente, más de la mitad son leves y no requieren tratamiento. ${ }^{3,8}$ Dentro de las complicaciones más relevantes destacamos la hemorragia y la incontinencia. La hemorragia es la complicación más frecuente (1-13 \%) y se asocia a dejar abierta la brecha rectal. Generalmente es leve y se detiene espontáneamente. En cuanto a la incontinencia, se ha reportado una incidencia de $10 \%$, de grado variable. Está dada fundamentalmente por la dilatación anal y colocación de la plataforma. ${ }^{9}$ Sin embargo, los últimos estudios han mostrado alteraciones principalmente manométricas sin traducción clínica. Se ha reportado tanto para TEM como para TAMIS. ${ }^{5}$

La dehiscencia de la línea de sutura y el desarrollo de abscesos perirrectales se observa en $5 \%$ de los casos. Este porcentaje aumenta en pacientes sometidos a neoadyuvancia. Las lesiones uretrales/vaginales se ven en $5,8 \% .{ }^{3}$ La perforación intraperitoneal en resecciones altas puede repararse por abordaje transanal o laparoscópico abdominal simultáneo. ${ }^{3}$ Otras complicaciones poco frecuentes son: estenosis anal, fístulas rectovaginales, lesiones vasculares y nerviosas, embolia gaseosa, neumoretroperitoneo, retención aguda de orina, dolor y fiebre. ${ }^{9}$

\section{CONCLUSIÓN}

Gracias a la accesibilidad y familiaridad de los materiales laparoscópicos para el cirujano, el TAMIS ha permitido extender la aplicación de los procedimientos endoscópicos por vía transanal, con una mejor calidad de resección que la técnica transanal convencional. Esto permite ampliar sus indicaciones, como en este caso, que se utilizó para la resección de un tumor rectal luego de una resección endoscópica insuficiente, permitiendo su estudio anatomopatológico completo y definiendo la conducta terapéutica.

\section{REFERENCIAS}

1. Moreira Grecco A, Zapata G, Bollo C, Morales R, Sarotto L. TAMIS: ¿Un nuevo estándar para el tratamiento de los pólipos de recto? Revisión de la bibliografía y reporte de nuestra experiencia. Rev Argent Coloproct 2019;30:1-11.

2. Buess G, Kipfmüller K, Hack D, Grüssner R, Heintz A, Junginger T. Technique of transanal endoscopic microsurgery. Surg Endosc 1988;2:71-5.

3. Rossi G. Relato oficial. Resecciones transanales: pasado, presente y futuro. Rev Argent Coloproct 2019;30:1-77.

4. Atallah S, Albert M, Larach S. Transanal minimally invasive surgery:
A giant leap forward. Surg Endosc 2010;24:2200-5.

5. Villanueva-Herrero JA, Alarcón-Bernes L, Jiménez-Bobadilla B. Cirugía endoscópica transanal: nuevas alternativas con instrumentos de cirugía laparoscópica convencional. Rev Mex Cirug Endosc 2011;12:174-79.

6. Qi Y, Stoddard D, Monson JRT. Indications and techniques of transanal endoscopic microsurgery (TEMS). J Gastrointest Surg 2011;15:1306-8.

7. Caselli G, Ocares M, Delgado C, Benavides C, Martin F, Madariaga J, et al. Cirugía transanal por puerto único para la resección local de 
lesiones de recto. Rev Chil Cir 2014; 66): 460-66.

8. Quinteros F, Thiruppathy K, Albert MR. Transanal minimally invasive surgery (TAMIS): Operative technique, pitfalls, and tips. In: Ross H, Lee S, Mutch M, Rivadeneira D, Steele S. (eds) Minimally invasive approaches to colon and rectal disease. Springer: New York, NY. 2015;25:283-91.

9. Heras MA, Cantero R. Cirugía transanal a través de puerto único (TAMIS). Revisión frente otras técnicas de excisión endoscópica de lesiones rectales. Rev Argent Coloproct 2013;2:55-60.

10. Arroyave MC, De Lacy B, Lacy AM. Transanal total mesorectal excision (TaTME) for rectal cancer: step by step description of the surgical technique for a two-team approach. Eur J Surg Oncol 2017;43: 502-5.

11. Waheed A, Miles A, Kelly J, Monson JRT, Motl JS, Albert M. Insufflation stabilization bag (ISB): a cost-effective approach for stable pneumorectum using a modified $\mathrm{CO} 2$ insufflation reservoir for TAMIS and taTME. Tech Coloproctol 2017;21:897-900.

12. Moreira Grecco A, Dip F, Sarotto L. Methylene blue TAMIS guided procedure facilitates adenomatous polyps resection. https:// www.sages.org/meetings/annual-meeting/abstracts-archive/ methylen-blue-tamis-guided-procedure-facilitates-adenomatouspolyps-resection/.

\section{COMENTARIO}

Se presenta un caso de resección transanal mininvasiva (TAMIS) de una cicatriz secuela de la exéresis endoscópica incompleta de una lesión del recto bajo informada como adenocarcinoma con infiltración submucosa de 4 mm de profundidad y márgenes de resección comprometidos. No se informa el grado de diferenciación, ni si existe o no invasión linfovascular o budding y aún cuando puede asumirse que es por su ausencia, debería exigirse al patólogo que lo explicite en su informe, dado que son otros factores histológicos que permiten definir a un carcinoma temprano como de bajo o alto riesgo de presentar tumor residual en la pared o ganglios mesorrectales positivos, luego de la exéresis local. No obstante, en este caso ya estaban presentes al menos dos factores de alto riesgo (margen de resección comprometido y profundidad de invasión tumoral $>1 \mathrm{~mm}$ en un pólipo sésil)..$^{1,2}$

Los autores deben ser felicitados por realizar la resección local exitosamente y sin complicaciones, obteniendo una pieza no fragmentada y completa para el análisis histopatológico y demostrando su pericia y experiencia con la técnica. Sin embargo, no era necesario realizar esta resección si tenemos en cuenta que el informe de la biopsia endoscópica ya indicaba la necesidad de una resección oncológica, no solo por el compromiso del margen que, de ser el único factor adverso, podría haberse corregido con la resección por TAMIS, sino esencialmente porque la profundidad de invasión submucosa de $4 \mathrm{~mm}$ define a una lesión como de alto riesgo. Hay consenso en que los carcinomas T1 con características histológicas de alto riesgo tienen altas tasas de recurrencia locorregional, semejantes a las de los T2. ${ }^{3}$

Según la presencia o no de los factores de riesgo, los cánceres tempranos presentan una tasa de ganglios mesorrectales positivos del 3 al $23 \%$, que también es más elevada en los ubicados en el tercio distal del recto. ${ }^{4}$ Estas adenopatías positivas pueden ser responsables de la recurrencia posterior a una resección local con márgenes libres. Lamentablemente, para predecirlas no se puede confiar en la resonancia magnética nuclear de alta resolución (RMN-AR) ni en la ecografía endorrectal (superior a la RMN-AR para el T), porque para estadificar el $\mathrm{N}$ la capacidad de ambos métodos es baja. Esto es debido probablemente a que en los pólipos malignos (T1) las adenopatías metastásicas suelen ser de menor tamaño (mediana 3,3 mm) que en los tumores más avanzados (T2: 6,2 mm; T3: 8,0 mm) y además los focos de cáncer son más pequeños (mediana $0,3 \mathrm{~mm}$ ), lo que hace dificultosa su identificación. ${ }^{5}$ Es así que aunque las guías de la Asociación de Coloproctología de Gran Bretaña e Irlanda recomiendan realizar una ecografía endorrectal en todos los tumores rectales polipoides antes de la resección local para evaluar el T, también establecen que no se debería confiar en este estudio ni en la RM-AR para evaluar la probabilidad del compromiso de los ganglios linfáticos. ${ }^{2}$

En esta paciente, sin comorbilidades, la evidencia indica la cirugía radical, más aún cuando a priori podría realizarse la conservación de esfínteres, porque a pesar de que el informe anatomopatológico de la resección por TAMIS no mostró lesión residual en la pared, no pueden descartarse adenopatías mesorrectales positivas en el segmento de implantación del pólipo. Afortunadamente y contra lo que podría suponerse, la resección local previa no afecta negativamente la realización de una proctectomía radical temprana, ni produce consecuencias oncológicas adversas. ${ }^{6}$

Rita L. O. Pastore

Hospital Juan A. Fernández, CABA, Argentina.

\section{REFERENCIAS}

1. Kitajima K, Fujimori T, Fujii S, Takeda J, Ohkura Y, Kawamata H, et al. Correlations between lymph node metastasis and depth of submucosal invasion in submucosal invasive colorectal carcinoma: a Japanese collaborative study. J Gastroenterol 2004;39:534-43.

2. Williams J, Pullan R, Hill J, Horgan P, Salmo E, Buchanan G, et al. Management of the malignant colorectal polyp: ACPGBI position statement. Colorectal Dis 2013;15 (Suppl. 2) 1-38.

3. Bach S, Hill J, Monson J, Simson J, Lane L, Merrie A, et al. A predictive model for local recurrence after transanal endoscopic microsurgery for 
rectal cancer. Br J Surg 2009;96:280-90.

4. Nascimbeni R, Burgart L, Nivatvongs S, Larson D. Risk of lymph node metastasis in T1 carcinoma of the colon and rectum. Dis Colon Rectum 2002;45:200-6.

5. Landmann R, Wong W, Hoepf J, Shia J, Guillem J, Temple L, et al. Limitations of early rectal cancer nodal staging may explain failure after local excision. Dis Colon Rectum 2007;50:1520-25.

6. Hahnloser D, Wolff B, Larson D, Ping J, Nivatvongs S. Immediate radical resection after local excision of rectal cancer: an oncologic compromise? Dis Colon Rectum 2005;48:429-37. 